\title{
Effect of tolvaptan on renal water and sodium excretion and blood pressure during nitric oxide inhibition: a dose- response study in healthy subjects
}

\author{
Safa Al Therwani", Jeppe Bakkestrøm Rosenbæk, Frank Holden Mose, Jesper Nørgaard Bech \\ and Erling Bjerregaard Pedersen
}

\begin{abstract}
Background: Tolvaptan is a selective vasopressin receptor antagonist. Nitric Oxide (NO) promotes renal water and sodium excretion, but the effect is unknown in the nephron's principal cells. In a dose-response study, we measured the effect of tolvaptan on renal handling of water and sodium and systemic hemodynamics, during baseline and NOinhibition with L-NMMA (L-NG-monomethyl-arginine).

Methods: In a randomized, placebo-controlled, double blind, cross over study, 15 healthy subjects received tolvaptan 15, 30 and $45 \mathrm{mg}$ or placebo. L-NMMA was given as a bolus followed by continuous infusion during $60 \mathrm{~min}$. We measured urine output $(\mathrm{UO})$, free water clearance $\left(\mathrm{C}_{\mathrm{H} 2 \mathrm{O}}\right)$, fractional excretion of sodium ( $\left(\mathrm{FE}_{\mathrm{Na}}\right)$, urinary aquaporin-2 channels (U-AQP2) and epithelial sodium channels ( $u$-ENaC $\mathrm{Y}$ ), plasma vasopressin ( $p$-AVP) and central blood pressure (cBP).

Results: During baseline, $\mathrm{FE}_{\mathrm{Na}}$ was unchanged. Tolvaptan decreased $\mathrm{u}-\mathrm{ENaC}_{\gamma}$ dose-dependently and increased p-AVP threefold, whereas U-AQP2 was unchanged. During tolvaptan with NO-inhibition, $\cup \mathrm{O}$ and $\mathrm{C}_{\mathrm{H} 2 \mathrm{O}}$ decreased dose-dependently. $\mathrm{FE}_{\mathrm{Na}}$ decreased dose-independently and $\mathrm{u}-\mathrm{ENaC}_{\gamma}$ remained unchanged. Central BP increased equally after all treatments.

Conclusions: During baseline, fractional excretion of sodium was unchanged. During tolvaptan with NO-inhibition, renal water excretion was reduced dose dependently, and renal sodium excretion was reduced unrelated to the dose, partly via an AVP dependent mechanism. Thus, tolvaptan antagonized the reduction in renal water and sodium excretion during NO-inhibition. Most likely, the lack of decrease in AQP2 excretion by tolvaptan could be attributed to a counteracting effect of the high level of p-AVP.
\end{abstract}

Trial registration: Clinical Trial no: NCT02078973. Registered 1 March 2014.

Keywords: Tolvaptan, Nitric oxide, ENaC, AQP2, Blood pressure, Vasoactive hormones

\section{Background}

Water and salt balance in the body is a result of complex interactions of hormones, especially vasopressin and the components in the renin-angiotensin-aldosterone system. Vasopressin sensitive aquaporin2 water channels and epithelial sodium channels are expressed in the principal cells in the collecting ducts [1-3]. Stimulation

\footnotetext{
* Correspondence: safatherwani@hotmail.com

University Clinic in Nephrology and Hypertension, Department of Medical Research, Holstebro Hospital, Aarhus University, Hospital Unit Jutland West, Laegaardvej 12, 7500 Holstebro, Denmark

of vasopressin receptors (V2) facilitates renal absorption of water and sodium [4-7]. This mechanism is antagonized by tolvaptan, a selective V2 receptor antagonist. NO may affect the renal urine concentration mechanism by membrane insertion of AQP2 in the collecting ducts principal cells [8-11]. However, it is debated whether NO inhibits or stimulates AQP-mediated water transport. In a previous study, we showed that NO promotes water excretion by a partly AVP dependent mechanism [12]. NO is synthesized from L-arginine by NO-synthase, an enzyme that is competitively inhibited by L-NMMA [13]. 
Systemic NO inhibition causes reduction in diuresis, natriuresis, and increases in blood pressure [14-16]. Several studies have documented the effect of selective V2 receptor antagonism on renal sodium and water excretion, but whether $\mathrm{NO}$ is involved in the response of the $\mathrm{V} 2$ receptors in the renal tubular and vascular function is not clarified. Recently, tolvaptan has been approved by the EMA for treatment of autosomal dominant polycystic kidney disease [17-21]. Also, tolvaptan is effective in conditions of dilutional hyponatremia, associated with congestive heart failure, cirrhosis and the syndrome of inappropriate secretion of antidiuretic hormone (SIADH) [22-26]. However, The European guideline on hyponatremia recommends against the use of tolvaptan in the clinical management of SIADH [27]. Thus, based on a most likely wide spread use of tolvap$\tan$ in the future, an in-depth knowledge is necessary regarding the effect of the tolvaptan on renal tubular function, vasoactive hormones and blood pressure. Our laboratory has previously investigated the effect of tolvaptan $15 \mathrm{mg}$ at baseline and during tolvaptan with $\mathrm{NO}$-inhibition in a randomized, placebo-controlled, double-blinded, crossover study [12]. We demonstrated a clear effect of tolvaptan $15 \mathrm{mg}$ at baseline and during tolvaptan with NO-inhibition on renal water excretion and the activity in $\mathrm{ENaC}$, but no knowledge is available about the effect of different doses regarding interaction between tolvaptan and NO-inhibition.

In the present study, we hypothesized 1. that tolvaptan increases water and sodium excretion in a dose-dependent manner, 2. that systemic NO-inhibition reduces this response dose-dependently, and 3. An increase in blood pressure and vasoactive hormones at baseline and during NO-inhibition counteracts tolvaptan's effect on the tubular sodium and water transport at least partially.

The purpose was to measure the effect tolvaptan on 1 . glomerular filtration rate and renal absorption of water and sodium (GFR ( ${ }^{51} \mathrm{Cr}$-EDTA-clearance), urinary output (UO), free water clearance $\left(\mathrm{C}_{\mathrm{H} 2 \mathrm{O}}\right)$, fractional excretion of sodium $\left(\mathrm{FE}_{\mathrm{Na}}\right)$, urinary excretion of aquaporin 2 (u-AQP2), and urinary excretion of a protein fragment of the epithelial sodium channel (u-ENaC ${ }_{\gamma}$ ), 2.blood pressure (brachial blood pressure $(\mathrm{Bbp})$, central $\mathrm{BP}(\mathrm{cBP})$, pulse wave velocity $(\mathrm{PW})$, augmentation index (AI)), and 3 . vasoactive hormones in plasma(vasopressin (p-AVP), angiotensinII ( $\mathrm{p}$-AngII), renin (PRC), and aldosterone (p-Aldo)), both at baseline and during tolvaptan with NO-inhibition in a randomized, placebocontrolled, double-blinded, crossover dose-response study of healthy volunteers.

\section{Methods}

\section{Subjects}

\section{Inclusion criteria}

Healthy non-smoking men and women, age between 18 and 40 year. and with BMI between 18.5 and $30 \mathrm{~kg} / \mathrm{m}^{2}$ were enrolled.

\section{Exclusion criteria}

Exclusion criteria were; arterial hypertension (bBP > $140 \mathrm{mmHg}$ systolic and/or $>90 \mathrm{mmHg}$ diastolic), history or clinical signs of neoplastic disease or disease of the heart, lungs, kidneys or endocrine organs, drug or alcohol abuse (I.e. > 14 units a week for women and 21 for men), medical treatment except oral contraceptives, pregnancy or breast-feeding, significant laboratory abnormalities in the screening test of blood samples (I.e. abnormal hemoglobin, white cell count, plasma sodium, plasma potassium, plasma creatinine, plasma alanine aminotransferase or serum cholesterol, plasma bilirubin and plasma albumin, and urine samples (I.e. albuminuria or glucosuria), abnormal electrocardiogram, blood donation less than 1 month prior to the study.

In fertile women contraceptive treatment must be used during and after the study period (I.e. oral contraceptives, spiral, depot injection of gestagen, sub dermal implantation, transdermal contraceptive patch and hormonal contraceptive vaginal ring).

Withdrawal criteria were development of one or more of the exclusion criteria, brachial blood pressure increase above 180/105 mmHg during infusion of L-NMMA, withdrawal of consent or lack of compliance.

\section{Study design}

The trial was performed as a randomized, doubleblinded, placebo- controlled, crossover, dose-response study in healthy subjects. The study consisted of four treatment periods, placebo or tolvaptan 15, 30 and $45 \mathrm{mg}$, with an intermediate wash- out period of at least 3 weeks to eliminate any carryover effects.

\section{Medications}

Tolvaptan (SAMSCA ${ }^{\oplus}$, Otsuka, Tokyo, Japan) 15, 30 and $45 \mathrm{mg}$ or placebo were coated in identical gelatine capsules and were orally administered at 8:00 AM.

L-NMMA (Bachem, Weil am Rhein, Germany) was dissolved in isotonic saline solution and given intravenously at 11:00 AM.

\section{Number of subjects}

Free water clearance $\left(\mathrm{C}_{\mathrm{H} 2 \mathrm{O}}\right)$ was used as the main effect variable. With a minimal relevant difference of $6 \mathrm{ml} / \mathrm{min}$ with an estimated standard deviation (SD) of $4 \mathrm{ml} / \mathrm{min}$, 12 subjects were needed using a level of significance of $5 \%$ and a statistical power of $90 \%$. Due to possible drop outs 15 subjects were included.

\section{Recruitment}

Healthy volunteers were recruited by advertising in public institutions and private companies. 


\section{Effect variables}

$\mathrm{C}_{\mathrm{H} 2 \mathrm{O}}$ was the primary effect variable. The secondary effect variables were 1) renal function $\left({ }^{51} \mathrm{Cr}\right.$-EDTA-clearance, $\mathrm{UO}, \mathrm{u}-\mathrm{AQP} 2, \mathrm{u}-\mathrm{ENaC}_{\gamma}, \mathrm{FE}_{\mathrm{Na}}$ ), 2) hemodynamics (bBP, cBP, PWV, AI and 3) vasoactive hormones (PRC, p-ANG II, p-Aldo, p- AVP).

\section{Diet}

Four days prior to each treatment period subjects consumed a standardized diet of $11.000 \mathrm{KJ} \mathrm{day}^{-1}$. The diet was delivered from our facilities and was composed of $15 \%$ proteins, $55 \%$ carbohydrates and 30\% fat following general dietary guidelines. The sodium content was $150 \mathrm{mmol} \mathrm{day}^{-1}$. No additional sodium or other spices was allowed. The daily fluid intake was $2.5 \mathrm{~L}$ including a maximum of two cups of coffee or tea. No alcohol or soft drink was allowed during the 4-day diet.

\section{Experimental procedure}

A 24-h urine was collected and a fasting period of $8 \mathrm{~h}$ was performed prior to each examination day. The four examinations were conducted at our facility from 7:45 AM to 1:00 PM. The procedures were identical on all examination days.

At 8:00 AM subjects were given placebo or tolvaptan 15,30 and $45 \mathrm{mg}$. An intravenous catheter was placed in each arm to collect blood samples and infuse ${ }^{51} \mathrm{Cr}$ EDTA.

At 8:00 AM and every $30 \mathrm{~min}$, an oral water load of $175 \mathrm{ml}$ was given.

BP was measured every $30 \mathrm{~min}$ from 8:30 AM to 1:00 PM. Based on results from a dose-finding study made by our laboratory in healthy subjects [15], a bolus of L-NMMA $4.5 \mathrm{mg} / \mathrm{kg}$ was given at 11:00 AM, followed by continuous infusion $(3 \mathrm{mg} / \mathrm{kg} / \mathrm{h})$ during $60 \mathrm{~min}$. During infusion of L-NMMA BP was measured every $5 \mathrm{~min}$, and every 15 min after infusion of L-NMMA.

Blood samples were drawn every 30 min from 8:30 to 1:00 PM and were analyzed for p-Na, p-osm, pcreatinine, p-albumin and $\mathrm{p}-{ }^{51} \mathrm{Cr}$-EDTA. Every $60 \mathrm{~min}$; at 11:00 AM (baseline), at 12:00 AM (after end of L-NMMA infusion), and at 1:00 PM (60 min after end of L-NMMA infusion), blood samples were drawn to measure PRC, p-Aldo, p-Ang II and p-AVP.

Every 30 min from 9:30 AM to 1:00 PM, urine samples were collected by voiding in standing or sitting position after collecting BP measurements and blood samples. Otherwise subjects were kept in a supine position in a temperature-controlled $\left(22-25{ }^{\circ} \mathrm{C}\right)$ and quiet room. Baseline period was mean of the first three clearance periods. The urine samples were analyzed for osmolality, creatinine, sodium, AQP2, $\mathrm{ENaC}_{\gamma}$ and ${ }^{51} \mathrm{Cr}$-EDTA.
Applanation tonometry with SpygmoCor was performed at 10:10 AM and at11: 40 AM to measure cBP, PVW and AI.

\section{Measurements \\ Renal function}

${ }^{51} \mathrm{Cr}$-EDTA-clearance was measured using the constant infusion clearance technique with 51Cr-EDTA as reference substance.

$\mathrm{C}_{\mathrm{H} 2 \mathrm{O}}$ was determined using the formula $\mathrm{C}_{\mathrm{H} 2 \mathrm{O}}=\mathrm{UO}$ $\mathrm{C}_{\text {osm }}$, where $\mathrm{C}_{\mathrm{osm}}$ is the osmolar clearance.

Clearance $(\mathrm{C})$ of substance $\mathrm{X}$ was calculated as $\mathrm{C}_{\mathrm{X}}=$ $\mathrm{U}_{\mathrm{X}} /\left(\mathrm{P}_{\mathrm{X}} \mathrm{x}\right.$ UO), where $\mathrm{U}_{\mathrm{X}}$ denotes concentration of $\mathrm{x}$ in urine, $P_{X}$ denotes concentration of $x$ in plasma, and $U O$ is urine excretion rate.

Fractional excretion of sodium was determined according to the following formula $\mathrm{FE}_{\mathrm{Na}}=\mathrm{C}_{\mathrm{Na}} /{ }^{51} \mathrm{Cr}-$ EDTA-clearance $x 100 \%$, where $C_{\mathrm{Na}}$ is sodium clearance.

\section{Urinary excretion of $A Q P 2$ and $E N a C_{Y}$}

Urine samples were kept frozen at $-20{ }^{\circ} \mathrm{C}$ until assayed. $\mathrm{U}-\mathrm{AQP} 2$ and $\mathrm{u}-\mathrm{ENaC}_{\gamma}$ were measured by radioimmunoassay as previously described [28-30]. Antibodies were raised in rabbits to synthetic peptide as previously described [12]. U-ENaC $\mathrm{C}_{\gamma}$ was measured by radioimmunoassay as previously described [29]. Antibodies were raised against the synthetic $\mathrm{ENaC}_{\gamma}$ peptide in rabbits and affinity purified as described previously $[12,31]$. The anti-AQP2 antibody was a gift from Soerens Nielsen, The Water and Salt Research Center, Institute of Anatomy, Aarhus University, Denmark.

\section{Vasoactive hormones in plasma}

Blood samples collected for measurements of vasoactive hormones were centrifuged and plasma was separated, and kept frozen until assayed as previously described [12]. PRC was determined by immunoradiometric assay as previously described [12]. Aldo was determined by RIA as previously described [12]. Ang II and AVP were extracted from plasma and then determined by radioimmunoassay $[12,32,33]$.

\section{Brachial and central blood pressure}

Brachial BP was measured using an oscillometer (Omron 705IT) and recorded as previously described [12]. Central BP, PWA and carotid-femoral PWV were measured using applanation tonometry (SphygmoCor ${ }^{\bullet} \mathrm{CPV}$ system $^{\circ}$, AtCor Medical, Sydney, Australia) as previously described [12].

\section{Routine analyses}

Sodium, glucose, albumin, and hemoglobin were measured by routine methods in Department of Clinical Biochemistry, Holstebro Hospital. 


\section{Statistics}

We performed statistical analyses by using IBM SPSS statistics version 20.0.0 (SPSS Inc., Chicago, IL, USA). General Linear Model Repeated Measures was used for comparison between and within subjects to test differences between placebo and tolvaptan treatment at baseline, during and after infusion of L-NMMA. One-way ANOVA was performed for comparison between treatment groups.

To compare differences between treatment groups at baseline, paired sample $t$-test was performed during and after infusion of L-NMMA. Statistical significance was at $<0.05$ in all analyses. Data with normal distribution are reported as means \pm SD or SEM. Non-parametric test was performed for data with non-normal distribution, and are reported as medians with 25th and 75th percentiles.

\section{Results}

\section{Demographics}

Twenty-seven healthy subjects were allocated to the trial. Twelve of the participants were excluded; three due to difficulties to gain intravenous access, two due to arterial hypertension and seven due to withdrawal of consent. Fifteen participants completed the study, three males and 12 females. Median age was $24 \pm 2$ years, weight $71.8 \pm 13.1 \mathrm{~kg}$, BMI $24 \pm 4 \mathrm{kgm}^{2}$. P-sodium $141 \pm$ $1.7 \mathrm{mmol} / \mathrm{L}, \mathrm{p}$-potassium $3.7 \pm 0.2 \mathrm{mmol} / \mathrm{L}$, p-creatinine $68.7 \pm 9.8 \mu \mathrm{mol} / \mathrm{L}$. Systolic brachial blood pressure (SBP) $119 \pm 10 \mathrm{mmHg}$, diastolic brachial blood pressure (DBP) $72 \pm 8 \mathrm{mmHg}$.

\section{Urine collection before the examination day}

Twenty-four hours urine samples were collected prior to every examination day. Values are shown in Additional file 1: Table S1. No differences were measured in UO, $\mathrm{C}_{\mathrm{H} 2 \mathrm{O}}, \mathrm{u}-\mathrm{AQP} 2, \mathrm{u}-\mathrm{Na}$ and $\mathrm{u}-\mathrm{K}$ between the treatment periods.

\section{${ }^{51} \mathrm{Cr}$-EDTA-clearance}

During tolvaptan with NO inhibition, ${ }^{51} \mathrm{Cr}$-EDTA-clearance did not change significantly. The relative changes in ${ }^{51} \mathrm{Cr}$-EDTA-clearance were non-significant in the four treatment arms (Table 1).

\section{Urinary output and free water clearance}

Absolute and relative values of $\mathrm{UO}$ and $\mathrm{C}_{\mathrm{H} 2 \mathrm{O}}$ are shown in Fig. 1b-c and Table 1.

During L-NMMA infusion 90-120 min, UO decreased significantly during all treatments (placebo: $3.0 \mathrm{ml} / \mathrm{min}$ (49\%); tolvaptan $15 \mathrm{mg}: 4.8 \mathrm{ml} / \mathrm{min}$ (46\%); tolvaptan $30 \mathrm{mg}$ : $4.6 \mathrm{ml} / \mathrm{min}$ (40\%); tolvaptan $45 \mathrm{mg}$ : $3.6 \mathrm{ml} / \mathrm{min}$ $(31 \%))$. As during baseline, the absolute level of UO remained lower during placebo than tolvaptan, and no significant differences were measured between the different doses of tolvaptan. The relative changes from baseline to L-NMMA infusion were the same during both placebo and tolvaptan treatment. However, a tendency to a dose response effect was seen both in the absolute and relative measurements. During the post infusion period 180-210 $\mathrm{min}$, UO increased during placebo to the same level as during baseline, but remained reduced to the same levels during the three doses of tolvaptan (placebo: $0.7 \mathrm{ml} / \mathrm{min}$ (6\%); tolvaptan $15 \mathrm{mg}: 4.1 \mathrm{ml} / \mathrm{min}$ (40\%); tolvaptan $30 \mathrm{mg}$ : $4.6 \mathrm{ml} / \mathrm{min}$ (39\%); tolvaptan $45 \mathrm{mg}: 3.1 \mathrm{ml} / \mathrm{min}(31 \%))$.

During L-NMMA infusion 90-120 min, $\mathrm{C}_{\mathrm{H} 2 \mathrm{O}} \mathrm{de}-$ creased during all treatments (placebo: $2.1 \mathrm{ml} / \mathrm{min}$ (57\%); tolvaptan $15 \mathrm{mg}: 4.3 \mathrm{ml} / \mathrm{min}$ (53\%); tolvaptan $30 \mathrm{mg}: 4.2 \mathrm{ml} / \mathrm{min}$ (38\%); tolvaptan $45 \mathrm{mg}: 3.3 \mathrm{ml} / \mathrm{min}$ (35\%)). The absolute level of $\mathrm{C}_{\mathrm{H} 2 \mathrm{O}}$ remained lower during placebo than tolvaptan treatment, and $\mathrm{C}_{\mathrm{H} 2 \mathrm{O}}$ decreased significantly more after tolvaptan $15 \mathrm{mg}$ compared to 30 and $45 \mathrm{mg}$ ( $p=0.034$ and 0.004 respectively). The relative changes from baseline to L-NMMA infusion did not deviate significantly between the four treatments, but a tendency to a dose response effect was measured with the lowest decrease in the highest tolvaptan dosis. During the post infusion period 180210 min, $C_{\mathrm{H} 2 \mathrm{O}}$ increased during placebo to the same level as during baseline, but remained reduced during tolvaptan treatment (placebo: $0.5 \mathrm{ml} / \mathrm{min}$ (0.5\%); tolvaptan $15 \mathrm{mg}: 4.2 \mathrm{ml} / \mathrm{min}$ (51\%)); tolvaptan $30 \mathrm{mg}$ : $4.2 \mathrm{ml} / \mathrm{min}$ (42\%); tolvaptan $45 \mathrm{mg}: 3.2 \mathrm{ml} / \mathrm{min}$ (31\%)). The difference in $\mathrm{C}_{\mathrm{H} 2 \mathrm{O}}$ between the three tolvaptan doses was non-significant but a tendency to a dose response effect was seen.

\section{Fractional excretion of sodium}

Absolute and relative values of $\mathrm{FE}_{\mathrm{Na}}$ are shown in Fig. 2e and Table 1.

During L-NMMA infusion 90-120 min, $\mathrm{FE}_{\mathrm{Na}}$ decreased significantly after placebo and tolvaptan $15 \mathrm{mg}$ (placebo: $0.37 \%$ (the relative decrease in percent: 25\%); tolvaptan $15 \mathrm{mg}$ : $0.20 \%$ (14\%); tolvaptan $30 \mathrm{mg}$ : $0.12 \%$ (15\%); tolvaptan $45 \mathrm{mg}: 0.14 \%$ (12\%)). However, $\mathrm{FE}_{\mathrm{Na}}$ decreased to the same level as after tolvaptan 30 and $45 \mathrm{mg}$ during the first $30 \mathrm{~min}$ of LNMMA infusion period 90-120 min. However, during the last LNMMA infusion period $120-150 \mathrm{~min}, \mathrm{FE}_{\mathrm{Na}}$ decreased to a significantly lesser extent after all tolvaptan doses compared with placebo (Table 1). During the post infusion period $180-210 \mathrm{~min}, \mathrm{FE}_{\mathrm{Na}}$ increased after all treatments, but to a much higher level after tolvaptan (placebo: $0.04 \%$ (the increase in percent; 4\%); tolvaptan $15 \mathrm{mg}$ : $0.36 \%$ (46\%); tolvaptan 30 mg:0.52\% (44\%); tolvaptan $45 \mathrm{mg}$ : 0.42\% (45\%)). 
Table 1 Effect of tolvaptan 15, 30 and $45 \mathrm{mg}$ at baseline, during and after NO-inhibition on the relative changes in GFR ( $\Delta^{51}$ CrEDTAclearance), urinary output $(\triangle U O)$, free water clearance $\left(\Delta C_{H 2 O}\right)$, urinary aquaporin 2 excretion rate $(\triangle A Q P 2)$, fractional excretion of sodium $\left(\triangle \mathrm{FE}_{\mathrm{Na}}\right)$ and urinary $\mathrm{ENaCY}$ excretion rate $(\Delta \mathrm{ENaC \gamma})$ in a randomized, placebo-controlled, double-blind, crossover, dose-response study of 15 healthy subjects

\begin{tabular}{|c|c|c|c|c|c|c|}
\hline \multirow[t]{2}{*}{ Periods } & \multirow{2}{*}{$\begin{array}{l}\text { Baseline } \\
\text { 0-90 min }\end{array}$} & \multicolumn{2}{|l|}{ L-NMMA } & \multicolumn{2}{|l|}{ Post infusion } & \multirow{2}{*}{$\begin{array}{l}p \\
\text { (GLM- } \\
\text { within) }\end{array}$} \\
\hline & & $90-120 \mathrm{~min}$ & $120-150 \mathrm{~min}$ & $150-180 \mathrm{~min}$ & $180-210 \mathrm{~min}$ & \\
\hline \multicolumn{7}{|c|}{$\overline{\Delta{ }^{51} \mathrm{Cr} \text {-EDTA-clearance (\%) }}$} \\
\hline Placebo & - & $-5 \pm 9$ & $-1 \pm 2$ & $-5 \pm 3$ & $-3 \pm 8$ & \multirow[t]{5}{*}{0.746} \\
\hline Tolvaptan 15 mg & - & $-1 \pm 7$ & $2 \pm 4$ & $-2 \pm 5$ & $1 \pm 5$ & \\
\hline Tolvaptan $30 \mathrm{mg}$ & - & $-5 \pm 7$ & $-3 \pm 7$ & $-3 \pm 6$ & $-4 \pm 7$ & \\
\hline Tolvaptan 45 mg & - & $1 \pm 6$ & $1 \pm 7$ & $-0.1 \pm 7$ & $-2 \pm 7$ & \\
\hline $\mathrm{p}$ (GLM between) & & & 0.447 & & & \\
\hline \multicolumn{7}{|l|}{$\Delta \cup O(\%)$} \\
\hline Placebo & - & $-49(-59 ;-13)$ & $-41(-49 ;-5)$ & $-26(-42 ; 5)$ & $-6(-27 ; 30)$ & \multirow[t]{6}{*}{$<0.0001$} \\
\hline Tolvaptan $15 \mathrm{mg}$ & - & $-46(-57 ;-34)$ & $-48(-51 ;-38)$ & $-45(-49 ;-39)$ & $-40(-47 ;-26)$ & \\
\hline Tolvaptan $30 \mathrm{mg}$ & - & $-40(-54 ;-32)$ & $-42(-55 ;-34)$ & $-47(-55 ;-28)$ & $-39(-44 ;-25)$ & \\
\hline Tolvaptan 45 mg & - & $-31(-38 ;-27)$ & $-42(-43 ;-31)$ & $-32(-41 ;-26)$ & $-31(-36 ;-24)$ & \\
\hline $\mathrm{p}$ (GLM between) & & & 0.002 & & & \\
\hline $\mathrm{p}$ (ANOVA) & - & 0.217 & 0.078 & 0.001 & $<0.0001$ & \\
\hline \multicolumn{7}{|l|}{$\Delta \mathrm{C}_{\mathrm{H} 2 \mathrm{O}}(\%)$} \\
\hline Placebo & - & $-57(-78 ;-25)$ & $-47(-63 ; 0)$ & $-31(-50 ; 14)$ & $-0.5(-31 ; 37)$ & \multirow[t]{6}{*}{$<0.0001$} \\
\hline Tolvaptan 15 mg & - & $-53(-66 ;-41)$ & $-56(-60 ;-39)$ & $-54(-66 ;-47)$ & $-51(-59 ;-46)$ & \\
\hline Tolvaptan 30 mg & - & $-38(-65 ;-33)$ & $-46(-66 ;-38)$ & $-51(-63 ;-35$ & $-42(-61 ;-31)$ & \\
\hline Tolvaptan 45 mg & - & $-35(-49 ;-30)$ & $-47(-57 ;-36)$ & $-33(-54 ;-30)$ & $-31(-46 ;-26)$ & \\
\hline $\mathrm{p}$ (GLM between) & & & 0.004 & & & \\
\hline$p$ (ANOVA) & - & 0.156 & 0.130 & 0.011 & 0.002 & \\
\hline \multicolumn{7}{|l|}{$\triangle \mathrm{AQP} 2(\%)$} \\
\hline Placebo & - & $-19(-29 ;-2)$ & $-21(-29 ;-18)$ & $-21(-28 ;-12)$ & $-13(-23 ;-3)$ & \multirow[t]{5}{*}{0.233} \\
\hline Tolvaptan 15 mg & - & $-18(-26 ;-7)$ & $-13(-18 ;-7)$ & $-9(-18 ;-2)$ & $-4(-18 ; 11)$ & \\
\hline Tolvaptan 30 mg & - & $-14(-26 ;-5)$ & $-19(-28 ;-11)$ & $-12(-24 ;-2)$ & $-4(-13 ; 6)$ & \\
\hline Tolvaptan 45 mg & - & $-9(-22 ; 3)$ & $-13(-22 ;-2)$ & $-13-23 ;-4)($ & $-2(-11 ; 6)$ & \\
\hline $\mathrm{p}$ (GLM between) & & & 0.094 & & & \\
\hline \multicolumn{7}{|l|}{$\Delta \mathrm{FE}_{\mathrm{Na}}(\%)$} \\
\hline Placebo & - & $-25(-33 ;-9)$ & $-33(-40 ;-28)$ & $-12(-30 ; 1)$ & $4(-11 ; 25)$ & \multirow[t]{12}{*}{0.433} \\
\hline Tolvaptan 15 mg & - & $-14(-28 ;-3)$ & $-17(-23 ; 8)$ & $17(-5 ; 34)$ & $46(0 ; 55)$ & \\
\hline Tolvaptan 30 mg & - & $-15(-18 ; 9)$ & $-10(-18 ; 9)$ & $13(0 ; 19)$ & $44(19 ; 61)$ & \\
\hline Tolvaptan 45 mg & - & $-12(-28 ; 4)$ & $-9(-26 ; 4)$ & $3(11 ; 64)$ & $45(19 ; 91)$ & \\
\hline $\mathrm{p}$ (GLM between) & & & 0.014 & & & \\
\hline$p$ (ANOVA) & - & 0.207 & 0.009 & 0.014 & 0.021 & \\
\hline \multicolumn{6}{|l|}{$\Delta \mathrm{ENaC}_{\gamma}(\%)$} & \\
\hline Placebo & - & $-11(-16 ;-7)$ & $-8(-20 ;-3)$ & $-11(-23 ;-3)$ & $2(-13 ; 18)$ & \\
\hline Tolvaptan 15 mg & - & $-9(-21 ;-2)$ & $-7(-15 ; 22)$ & $5(-8 ; 13)$ & $6(-7 ;-21)$ & \\
\hline Tolvaptan 30 mg & - & $-3(-13 ; 3)$ & $-5(-10 ; 5)$ & $3(-1 ; 13)$ & $8(-8 ; 19)$ & \\
\hline Tolvaptan 45 mg & - & $-13(-21 ; 3)$ & $-5(-10 ; 6)$ & $-2(-8 ; 10)$ & $13(-7 ; 18)$ & \\
\hline $\mathrm{p}$ (Friedman) & - & 0.564 & 0.392 & 0.008 & 0.031 & \\
\hline
\end{tabular}

Data are presented as mean \pm SD or medians with $25^{\text {th }}$ and $75^{\text {th }}$ percentiles in parentheses. General linear model (GLM) with repeated measures was performed for comparison within and between groups or Friedman test for comparison between groups. One-way ANOVA was used to test differences between tolvaptan 15,30 and $45 \mathrm{mg}$ vs placebo. Paired $t$-test was used for comparison between the three tolvaptan doses at baseline period (0-90 min), L-NMMA period (90-150 $\mathrm{min}$ ) and post infusion period (150-210 $\mathrm{min}$ ); the significance levels are listed under the result section 

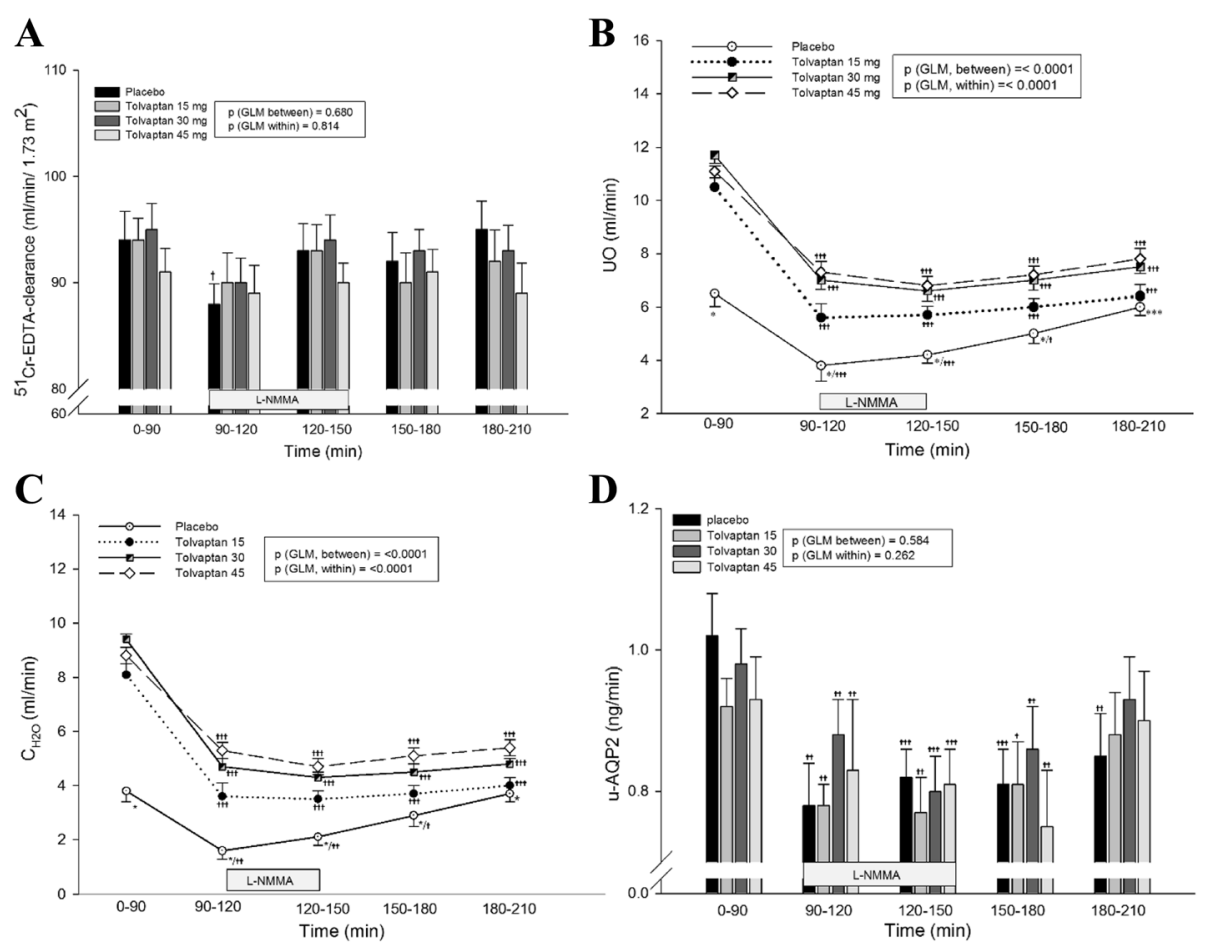

Fig. 1 Effect of tolvaptan 15, 30 and $45 \mathrm{mg}$ at baseline, during and after NO-inhibition on GFR ( ${ }^{51}$ Cr-EDTA-clearance) (a), UO (b), $\mathrm{C}_{\mathrm{H} 2 \mathrm{O}}(\mathbf{c})$ and U-AQP2 (d). Data are presented as mean \pm SEM. General linear model (GLM) with repeated measures was performed for comparison within and between groups. One-way ANOVA $\left({ }^{*}\right)$ was used to test differences between tolvaptan 15,30 and $45 \mathrm{mg}$ vs placebo. Paired $t$-test $(\alpha / \beta / \gamma)$ was used for comparison of infusion period (90-150 min) vs baseline period (0-90 min) and post infusion period (150-210 min) vs baseline period. $t p<$; 0.05 ; + t $p<0.001 ; *$ t†† $p<0.0001$. Paired t-test was used for comparison between the three tolvaptan doses at baseline period (0-90 min), L-NMMA infusion period (90-150 $\mathrm{min}$ ) and post infusion period (150-210 min); the significance levels are listed under the result section

No dose response pattern was seen either in the absolute or relative measurements of $\mathrm{FE}_{\mathrm{Na}}$.

\section{U-AQP2 and U-ENaC}

During tolvaptan with NO-inhibition 90-120 min, u-AQP2 decreased to the same level within all treatment arms. The same response was observed in the relative changes. During the post infusion period $180-210 \mathrm{~min}, \mathrm{u}-\mathrm{AQP} 2$ remained reduced during placebo treatment, but had increased to baseline level during all tolvaptan doses. No dose response pattern was seen, in either the absolute or relative measurements of $\mathrm{u}-\mathrm{AQP} 2$ (Fig. 1d and Table 1).
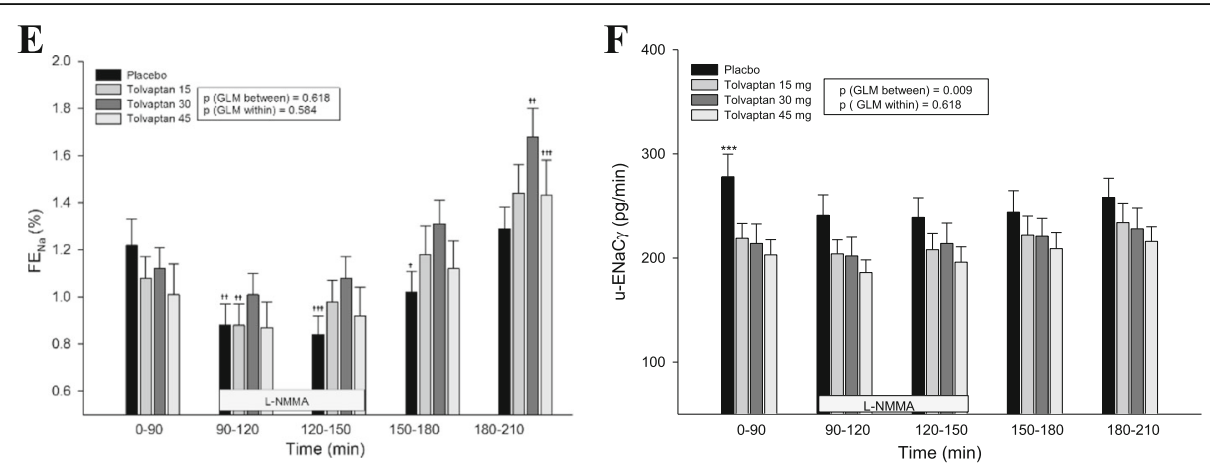

Fig. 2 Effect of tolvaptan 15, 30 and $45 \mathrm{mg}$ at baseline, during and after NO-inhibition on $\mathrm{FE}_{\mathrm{Na}}$ (e) and $\mathrm{u}$-ENaCY (f). Data are presented as mean \pm SEM. General linear model (GLM) with repeated measures was performed for comparison within and between groups. One-way ANOVA $(*)$ was used to test differences between tolvaptan 15, 30 and $45 \mathrm{mg}$ vs placebo. Paired $t$-test ( $\alpha / \beta / \gamma)$ was used for comparison of infusion period ( $90-150 \mathrm{~min})$ vs

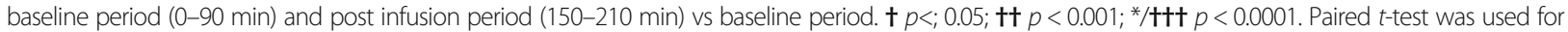
comparison between the three tolvaptan doses at baseline period (0-90 min), L-NMMA infusion period (90-150 min) and post infusion period (150-210 min); the significance levels are listed under the result section 
During tolvaptan with NO-inhibition 90-120 min, $\mathrm{u}-\mathrm{ENaC}_{\gamma}$ decreased significantly after placebo, but during tolvaptan treatment no significant differences existed between the placebo group and the three tolvaptan doses either in absolute or relative measurements (Fig. $2 \mathrm{f}$ and Table 1). During the post infusion period 180-210 min, u-ENaC ${ }_{\gamma}$ was unchanged compared to baseline measurement both during placebo and tolvaptan treatment. A significant difference was measured in the relative values with a dose response pattern and the highest increase during the highest tolvaptan dosis (Table 1).

\section{Plasma sodium and plasma osmolarity}

At baseline, $\mathrm{p}-\mathrm{Na}$ and $\mathrm{p}$-osm were significantly higher and similar after tolvaptan 15, 30 and $45 \mathrm{mg}$ compared to placebo and this response was sustained during tolvaptan with NO-inhibition (Additional file 2: Table S2).

\section{Vasoactive hormones}

During tolvaptan with NO-inhibition, none of the vasoactive hormones were affected a part from the sustained 3- fold increase in p-AVP after all three tolvaptan doses (Additional file 3: Table S3).

\section{Brachial blood pressure}

During baseline conditions, SBP, DBP and pulse rate were the same within all treatments (Additional file 4: Table S4).

In response to tolvaptan with NO-inhibition, DBP increased after placebo, but remained constant after tolvaptan 15, 30 and $45 \mathrm{mg}$. Otherwise no differences were measured in SBP and pulse rate.

\section{Central blood pressure}

At baseline, PWV, AI, CSBP and CDBP were identical after tolvaptan 15, 30 and $45 \mathrm{mg}$ versus placebo. During tolvaptan with NO-inhibition, CSBP and CDBP increased equally after all treatments (Table 2). PWV was not affected by L-NMMA infusion after placebo whereas a similar increase after tolvaptan 15, 30 and $45 \mathrm{mg}$ was measured, but only to the same level observed after placebo. Tolvaptan 15 and $30 \mathrm{mg}$ did not change AI. The value of AI was higher after tolvaptan $45 \mathrm{mg}$. However, no differences were measured between treatment groups.

\section{Water and sodium excretion, BP and vasoactive hormones} during baseline

${ }^{51} \mathrm{Cr}$-EDTA-clearance was similar after placebo and tolvaptan (Fig. 1a).

UO was significantly lower during placebo than tolvaptan treatment (Fig. 1b). UO was lower after tolvaptan $15 \mathrm{mg}$ compared with tolvaptan $30 \mathrm{mg}(p=0.005)$, while
Table 2 Effect of tolvaptan 15, 30 and $45 \mathrm{mg}$ at baseline, during and after NO-inhibition on pulse wave velocity (PW), augmentation index (Al), central diastolic and systolic blood pressure (CBDP and (SBP) in a randomized, placebo-controlled, double-blind, crossover, dose-response study of 15 healthy subjects

\begin{tabular}{|c|c|c|}
\hline & $\begin{array}{l}\text { Prior to L-NMMA } \\
\text { infusion (at } 70 \mathrm{~min} \text { ) }\end{array}$ & $\begin{array}{l}\text { During L-NMMA } \\
\text { infusion (at } 130 \mathrm{~min} \text { ) }\end{array}$ \\
\hline \multicolumn{3}{|l|}{$\mathrm{PWV}(\mathrm{m} / \mathrm{s})$} \\
\hline Placebo & $5.2 \pm 0.5$ & $5.4 \pm 0.5$ \\
\hline Tolvaptan 15 mg & $5.0 \pm 0.5$ & $5.4 \pm 0.6^{* *}$ \\
\hline Tolvaptan 30 mg & $5.2 \pm 0.6$ & $5.5 \pm 0.6^{* *}$ \\
\hline Tolvaptan 45 mg & $5.2 \pm 0.3$ & $5.5 \pm 0.4^{* *}$ \\
\hline $\mathrm{p}$ (ANOVA) & 0.518 & 0.851 \\
\hline \multicolumn{3}{|l|}{$\mathrm{Al}$} \\
\hline Placebo & $-0.7 \pm 13.8$ & $0.1 \pm 15.1$ \\
\hline Tolvaptan 15 mg & $-1.5 \pm 12.5$ & $-1.2 \pm 17.3$ \\
\hline Tolvaptan 30 mg & $-0.4 \pm 11.8$ & $2.5 \pm 17.8$ \\
\hline Tolvaptan 45 mg & $-3.3 \pm 15.8$ & $1.6 \pm 12.0^{*}$ \\
\hline$p($ ANOVA) & 0.968 & 0.961 \\
\hline \multicolumn{3}{|l|}{ CSBP } \\
\hline Placebo & $97 \pm 9$ & $100 \pm 8^{* *}$ \\
\hline Tolvaptan 15 mg & $97 \pm 9$ & $101 \pm 10^{*}$ \\
\hline Tolvaptan 30 mg & $96 \pm 9$ & $102 \pm 9^{* *}$ \\
\hline Tolvaptan 45 mg & $96 \pm 8$ & $102 \pm 6^{* *}$ \\
\hline$p($ ANOVA) & 0.989 & 0.952 \\
\hline \multicolumn{3}{|l|}{ CDBP } \\
\hline Placebo & $63 \pm 7$ & $68 \pm 6^{* * *}$ \\
\hline Tolvaptan 15 mg & $63 \pm 7$ & $68 \pm 9^{* *}$ \\
\hline Tolvaptan 30 mg & $61 \pm 6$ & $68 \pm 7^{* * *}$ \\
\hline Tolvaptan 45 mg & $62 \pm 5$ & $68 \pm 6^{* *}$ \\
\hline$p($ ANOVA) & 0.936 & 0.998 \\
\hline
\end{tabular}

Data are presented as mean \pm SD. One-way ANOVA was performed to test differences between treatments groups. Paired $t$-test $\left({ }^{*}\right)$ was used for comparison of data during L-NMMA infusion vs prior to L-NMMA infusion ${ }^{*} p<0.05 ;{ }^{* *} p<0.001 ;{ }^{* * *} p<0.0001$

no difference was measured between tolvaptan 30 and $45 \mathrm{mg}$. $\mathrm{C}_{\mathrm{H} 2 \mathrm{O}}$ was significantly lower during placebo than tolvaptan treatment. $\mathrm{C}_{\mathrm{H} 2 \mathrm{O}}$ increased significantly more after tolvaptan $30 \mathrm{mg}$ compared with $15 \mathrm{mg}(p=0.004)$, but no further increase was measured after tolvaptan $45 \mathrm{mg}$ (Fig. 1c).

$\mathrm{FE}_{\mathrm{Na}}$ was the same after all treatments. No significant difference was measured between the three tolvaptan doses (Fig. 2e).

U-AQP2 was similar after tolvaptan 15,30 and $45 \mathrm{mg}$ or placebo (Fig. 1d). U-ENaC $\mathrm{E}_{\gamma}$ was significantly higher after placebo than after tolvaptan treatment, and a doseresponse effect was measured with the lowest level of $\mathrm{u}-\mathrm{ENaC} \gamma$ during the highest tolvaptan dosis (Fig. 2f). 
PRC, p-ANGII and p-Aldo were the same after tolvaptan 15, 30 and $45 \mathrm{mg}$ compared with placebo (Additional file 3: Table S3). A highly significant and non-dose dependent 3-fold increase in p-AVP was measured after all three doses of tolvaptan (Fig. 3). No difference was measured between the three tolvaptan doses $(p=0.645)$.

\section{Discussion}

In the present dose-response study, we investigated short-term effects of tolvaptan on renal tubular function, vasoactive hormones and systemic hemodynamics during basal conditions and during tolvaptan with NO-inhibition in healthy subject. The present study is a continuation and expansion of a previous randomized, double-blinded, placebo-controlled crossover, singledose study conducted by our laboratory [12].

At baseline, tolvaptan increased renal water excretion dose-dependently up to $30 \mathrm{mg}$, without affecting sodium excretion. During L-NMMA infusion, tolvaptan decreased water excretion dose-dependently, whereas sodium excretion decreased independently of the dose. In the post infusion period, water excretion remained reduced during all tolvaptan doses, but increased during placebo. Tolvaptan increased sodium excretion to a higher level.

Recent dose-response trials have shown that oral administration of tolvaptan $30 \mathrm{mg}$ led to a fast increase in the effective concentration in plasma. This increase was however followed by a fast decline due to tolvaptan's short half-life of approximately $6-8 \mathrm{~h}[34,35]$. The absolute bioavailability of tolvaptan is approximately $56 \%$ and a plasma concentration peak $(300 \mathrm{ng} / \mathrm{ml})$ is obtained $2-4 \mathrm{~h}$ post-dosing. Based on the pharmacological profile

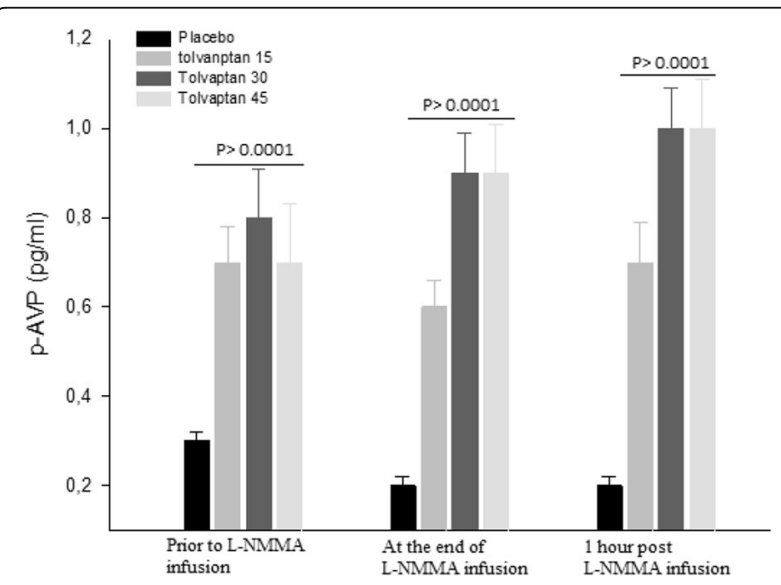

Fig. 3 Effect of tolvaptan 15, 30 and $45 \mathrm{mg}$ on p-AVP at baseline, during and after NO-inhibition. Data are depicted as mean \pm SEM. Friedman test was used for comparison between treatment groups prior to L-NMMA infusion, at the end of L-NMMA infusion and $1 \mathrm{~h}$ after the end of L-NMMA infusion of tolvaptan, we think that oral administration of tolvap$\tan$ at 8:00 AM (approximately $3 \mathrm{~h}$ prior to L-NMMA infusion) was the optimal time. The results from our previous study of tolvaptan $15 \mathrm{mg}$ confirmed a significant increase in $\mathrm{OU}$ and $\mathrm{C}_{\mathrm{H} 2 \mathrm{O}}$ that was measurable within approximately $2-3 \mathrm{~h}$ post-dose [12].

In the present trial, tolvaptan increased $\mathrm{UO}$ and $\mathrm{C}_{\mathrm{H} 2 \mathrm{O}}$ dose-dependently when tolvaptan dose was ascended from 15 to $30 \mathrm{mg}$ during baseline, but no further increase was measured after tolvaptan $45 \mathrm{mg}$. This observation does not exclude that a much higher dose might further increase renal water excretion. In addition, a longer observation time might be needed to obtain a further increase in $\mathrm{UO}$ and $\mathrm{C}_{\mathrm{H} 2 \mathrm{O}}$. Several of the participants reported significant and frequent diuresis after they have left our facilities. In previous clinical studies, using much higher tolvaptan doses ranging from 60 to $480 \mathrm{mg}$, tolvaptan increased $\mathrm{C}_{\mathrm{H} 2 \mathrm{O}}$ in a dose-independent relationship and UO in a dose-dependent relationship [35, 36]. In these studies, $\mathrm{UO}$ and $\mathrm{C}_{\mathrm{H} 2 \mathrm{O}}$ were collected and measured over a longer observation period around 24-h and up to $144 \mathrm{~h}$ post-dose.

During L-NMMA infusion, $\mathrm{UO}$ and $\mathrm{C}_{\mathrm{H} 2 \mathrm{O}}$ declined in all treatment arms, confirming the well-known antidiuretic effect by blocking the diuretic action of NO. However, UO remained approximately $30-50 \%$ higher after tolvaptan, and p-AVP also remained at the high level and unchanged from baseline. The degree of decline in $\mathrm{C}_{\mathrm{H} 2 \mathrm{O}}$ tended to be dependent of the tolvaptan dose, as $\mathrm{C}_{\mathrm{H} 2 \mathrm{O}}$ fell less after tolvaptan 30 and $45 \mathrm{mg}$ than after $15 \mathrm{mg}$. In the post infusion period, $\mathrm{UO}$ and $\mathrm{C}_{\mathrm{H} 2 \mathrm{O}}$ remained reduced to the same levels during the three tolvaptan doses. This may reflect an interaction between the V2 receptor system and the NO system, expressed as a higher level of water excretion after tolvaptan. Thus, tolvaptan partly antagonized the antidiuretic action of L-NMMA. This is in agreement with NO having a stimulating effect on renal water excretion in the renal principal cells that seemed to be partly AVP-dependent. Our results are, however, not in agreement with the findings observed in our previous clinical study of tolvaptan $15 \mathrm{mg}$, in which we showed that tolvaptan potentiated the antidiuretic action of L-NMMA [12]. A possible explanation of this discrepancy could be that tolvaptan was given $2 \mathrm{~h}$ earlier, at 6:00 AM versus 8:00 AM in the present study. Therefore, tolvaptan exerted its effect over a longer period before the L-NMMA intervention.

Tolvaptan prevented fusion of cytoplasmic vesicles containing AQP2 to the apical membrane as response to activation of V2 receptors by AVP [1,2]. As a result, the activation rate of AQP2 is declined. This response is biochemically reflected as a decline in the excretion of AQP2 into the urine [28-30]. Increased $\mathrm{u}-\mathrm{AQP} 2$ reflects 
increased water transport from the tubular lumen to the intracellular space via the aquaporin 2 water channels, located in the principal cells in the distal part of the nephron $[28,30]$. Surprisingly, u-AQP2 did not change after oral administration of tolvaptan, not even in response to the highest dose of tolvaptan. We measured a similar response in our previous study of tolvaptan $15 \mathrm{mg}$ [12]. Most likely, this could be explained by the measured 3fold increase in plasma AVP. The increase in AVP exerts a competitive inhibition on the $\mathrm{V} 2$ receptor, thereby interfering with tolvaptan's binding to the $\mathrm{V} 2$ receptor. Alternatively, the baseline period could be too short to reflect the expected decline in $\mathrm{u}-\mathrm{AQP} 2$ after oral administration of tolvaptan.

At baseline, $\mathrm{FE}_{\mathrm{Na}}$ was identical after all treatments, whereas $\mathrm{u}-\mathrm{ENaC}_{\gamma}$ decreased dose-independently after tolvaptan. Several experimental and clinical trials have appointed the AVP-V2R-ENaC $C_{\gamma}$ axis as an essential physiological mechanism in the final regulation of sodium excretion [6, 37]. Our findings are in agreement with these results, as we measured a reduced and similar baseline level of $\mathrm{u}-\mathrm{ENaC}_{\gamma}$ after all tolvaptan doses. There was a clear tendency to a dose-response pattern with the lowest $\mathrm{u}-\mathrm{ENaC}_{\gamma}$ at the highest dosis of tolvaptan. However, we did not measure an increase in $\mathrm{FE}_{\mathrm{Na}}$. This observation could be explained by the primarily aquaretic effect of selective V2R antagonism that is shown to be approximately 4 times the natriuretic effect [6].

During tolvaptan with $\mathrm{NO}$-inhibition, $\mathrm{FE}_{\mathrm{Na}}$ declined less pronouncedly after tolvaptan and the degree of the decline was not dependent of tolvaptan dosis. The sodium transport via the epithelial sodium channels is supposed to be reflected by the level of urinary excretion of a protein fraction from the channels $\left(\mathrm{u}-\mathrm{ENaC}_{\gamma}\right)[29,38]$. $\mathrm{U}-\mathrm{ENaC}_{\mathrm{\gamma}}$ decreased after placebo and remained unchanged, but at a lower level after tolvaptan treatment. In the post infusion period, $\mathrm{FE}_{\mathrm{Na}}$ increased more and dose-independently after tolvaptan. $\mathrm{U}^{-\mathrm{ENaC}_{\gamma}}$ also increased, but with a dose response relationship and the highest increase was measured during tolvaptan $45 \mathrm{mg}$. This observation was different from our previous singledose study of tolvaptan, where $\mathrm{u}-\mathrm{ENaC}_{\gamma}$ increased after NO-inhibition and remained higher in the post infusion period. This in turn, could also be attributed to the $2 \mathrm{~h}$ longer reaction time for tolvaptan in the first study [12], during which the effective concentration in plasma may have been lowered owing tolvaptan's relatively short half-life. Therefore, a lesser effect remained when L-NMMA was administered. Thus, tolvaptan inhibited the antinatriuretic effect of systemic NO-inhibition partially via an AVP-dependent pathway, and the degree of inhibition followed a dose-independent relationship.

We measured a significant and three-fold non-dosedependent increase in p-AVP after all three tolvaptan doses. Endogenous AVP release appeared to reach a plateau at a tolvaptan dose of $15 \mathrm{mg}$. This compensatory release of AVP due to treatment with V2 receptor antagonist is well documented [26]. However, the absence of a dose-response pattern in vasopressin has previously been demonstrated during tolvaptan treatment. Also, this response in plasma AVP was not affected by inhibition of systemic NO after all treatments. Thus, NO did not seem to play a role in the AVP releasing mechanism. Our results are in agreement with previous studies $[6,12]$.

Brachial and central BP remained unaffected even with an approximate 3-fold increase in p-AVP after tolvaptan 15, 30 amd $45 \mathrm{mg}$. Applanation tonometry was also performed under standardized conditions. The method is described and evaluated elsewhere [39]. Inhibition of systemic NO synthesis caused an increase in cBP, but to the same level within all treatments. In addition to its aquaretic effect, tolvaptan is believed to exert a pressor effect via the V1a receptors, localized in the vascular smooth muscle cells, as a consequence of compensatory increased endogenous AVP release. In turn, AVP may activate the V1a receptors at high plasma levels [6]. However, we demonstrated that an increase in tolvaptan dose from 15 to $45 \mathrm{mg}$ had no effect on bBP and cBP. This is in good agreement with the fact that we did not measure changes in P-AngII, PRC or P-Aldo after any of the three tolvaptan doses. In other words, we could not reject the null hypothesis, saying that tolvaptan would not change blood pressure using different doses of tolvaptan.

During baseline conditions, we measured a non-dose dependent increase in $\mathrm{p}-\mathrm{Na}$ and $\mathrm{p}$-osm after tolvaptan. Plasma sodium and $\mathrm{p}$-osm remained $141 \mathrm{mmol} / \mathrm{L}$ and $288 \mathrm{mosm} / \mathrm{kg}$ respectively, even after tolvaptan $45 \mathrm{mg}$. Most likely, the response in $\mathrm{p}-\mathrm{Na}$ and $\mathrm{p}$-osm must be due to increased renal water excretion and consequently decline in the extracellular volume. V2 receptor antagonism has previously been shown to increase $\mathrm{p}-\mathrm{Na}$ and p-osm in a non-dose-response manner [12, 34, 36]. The abovementioned parameters were not affected by $\mathrm{NO}$ inhibition. The results are in agreement with a previous clinical study reported by our laboratory [12].

\section{Strengths and limitations}

The major strengths of our study are a combination of the study design as randomized, placebo-controlled, double-blinded, crossover trial, and the standardized diet and fluid intake to avoid any confounding of the results. It might be considered as a weakness of the study that the assumption of abrogated systemic NO synthesis was exclusively based on the measurement of the response in ${ }^{51} \mathrm{Cr}$-EDTA-clearance, $\mathrm{C}_{\mathrm{H} 2 \mathrm{O}}, \mathrm{UO}, \mathrm{u}-\mathrm{AQP}_{2}, \mathrm{FE}_{\mathrm{Na}}$ and mean arterial blood pressure. A measurement of nitrate or nitrite in plasma or urine could have documented the 
NO inhibition directly, but this method is difficult, and according to our experience not as reliable as the methods used in the present study.

Emerging data indicate that the regulated protein fractions of $\mathrm{AQP} 2$ and $\mathrm{ENaC}_{\gamma}$ are excreted as urinary exosomes. These fraction were not isolated and analyzed in the present study.

We cannot exclude possible gender differences in the parameters studied due to female predominance.

\section{Conclusions}

During baseline conditions, tolvaptan increased renal water excretion dose-dependently, whereas tubular handling of sodium was unchanged. During tolvaptan with NO-inhibition, renal water excretion was reduced dose-dependently, and renal sodium excretion non-dose-dependently partly via an AVP-dependent mechanism. The lack of decrease in $\mathrm{u}$ AQP2 by tolvaptan could be explained by a counteracting effect of elevated vasopressin in plasma.

\section{Additional files}

Additional file 1: Table S1. Urine output, free water clearance $\left(\mathrm{C}_{\mathrm{H} 2 \mathrm{O}}\right)$, urinary AQP2 excretion per minute (u-AQP2), urinary sodium excretion ( $\mathrm{u}-\mathrm{Na}$ ) and urinary potassium excretion $(\mathrm{u}-\mathrm{K})$ during 24-h urine collection in a randomised, placebo-controlled, double-blind, crossover, dose-response study of 15 healthy subjects. Values are means with \pm SD. One-way ANOVA was used for comparison between groups. (PDF $9 \mathrm{~kb}$ )

Additional file 2: Table S2. Effect of tolvaptan 15, 30 and $45 \mathrm{mg}$ at baseline, during and after NO-inhibition on plasma concentration of sodium and plasma osmolality in a randomized, placebo-controlled, double-blind, crossover, dose-response study of 15 healthy subjects. Data are presented as mean \pm SD. General linear model (GLM) with repeated measurements was performed for comparison within and between groups. One-way ANOVA was used to test differences between tolvaptan 15,30 and $45 \mathrm{mg}$ vs placebo. Paired t-test was performed for comparison of infusion period (90-150 min) vs baseline period (0-90 $\mathrm{min}$ ), and post infusion period (150-210 min) vs baseline period. (PDF $84 \mathrm{~kb}$ )

Additional file 3: Table S3. Effect of tolvaptan 15, 30 and $45 \mathrm{mg}$ at baseline, during and after NO-inhibition on plasma concentrations of renin (PRC), angiotensin II (P-Angll) and aldosterone (P-aldo) in a randomized, placebo-controlled, double-blind, crossover, dose-response study of 15 healthy subjects. Data are presented as mean \pm SD. General linear model (GLM) with repeated measures was performed for comparison within and between groups. Paired $t$-test was used for comparison between L-NMMA infusion period (at the end of L-NMMA infusion period) vs baseline (prior to L-NMMA infusion period) and at baseline vs post infusion period ( $1 \mathrm{~h}$ after L-NMMA infusion period) vs baseline period. One-way ANOVA was performed to test differences between treatment groups. (PDF $14 \mathrm{~kb}$ )

Additional file 4: Table S4. Effect of tolvaptan 15, 30 and $45 \mathrm{mg}$ at baseline, during and after NO-inhibition on brachial systolic blood pressure (SBP), diastolic blood pressure (DBP) and pulse rate in a randomized, placebo-controlled, double-blind, crossover, dose-response study of 15 healthy subjects. Data are presented as mean \pm SD. General linear model (GLM) with repeated measures was performed for comparison within and between treatment groups. Paired $t$-test was used for comparison of infusion period (at the beginning of LNMMA infusion period/at the end of LNMMA infusion period) vs baseline (prior to LNMMA infusion period) and LNMMA infusion period vs post infusion period (30 min after the end of LMMMA infusion period/60 min after the end of LNMMA infusion period). ${ }^{*} p<0.05$. One-way ANOVA was performed to test differences between treatment groups. (PDF $356 \mathrm{~kb}$ )

\section{Abbreviations}

Al: Augmentation index; bBP: brachial blood pressure; cBP: central blood pressure; $\mathrm{C}_{\mathrm{H} 2 \mathrm{O}}$ : Free water clearance; EMA: European Medicines Agency:; $\mathrm{FE}_{\mathrm{Na}}$ : Fractional excretion of sodium; GFR $\left({ }^{51} \mathrm{Cr}\right.$-EDTA-clearance): Glomerular filtration rate; L-NMMA: L-NG-monomethyl-arginine; NO: Nitric Oxide; p-Aldo: plasma aldosterone; p-Angll: plasma angiotensinll; p-AVP: plasma vasopressin; PRC: Plasma concentrations of rennin; PW: Pulse wave velocity; U-AQP2: urinary aquaporin-2 channels; u-ENaCy: urinary epithelial sodium channels; UO: Urine output

\section{Acknowledgement}

We thank our laboratory technicians, including Anne Mette Ravn, Henriette Vorup Simonsen and Kirsten Nyggard for their skillful assistance. We thank Otsuka for a research grant to the study.

\section{Funding}

The study was supported by grants from The Research Foundation of Central Denmark Region.

\section{Availability of data and materials}

The datasets used/or analyzed during the current study available from the corresponding author on reasonable request.

\section{Authors' contributions}

SAT participated in the coordination of the study, recruitment of subjects and performed statistical analysis. SAT and JBR performed the experiments. EBP conceived of the study, participated in the design of the study and was responsible for the laboratory analyses. SAT, JBR, FHM, JNB and EBP wrote and edited the manuscript. All authors read and approved the final manuscript.

\section{Competing interests}

The authors declare that they have no competing interests.

\section{Consent for publication}

Not applicable.

\section{Ethics approval and consent to participate}

This study was approved by The Central Denmark Region Committees on Health Research Ethics (Clinical Trial no: NCT02078973). The study was conducted in conformity with the principles of the declaration of Helsinki. Written informed consent was obtained from all subjects.

\section{Publisher's Note}

Springer Nature remains neutral with regard to jurisdictional claims in published maps and institutional affiliations.

Received: 28 November 2016 Accepted: 7 March 2017

Published online: 13 March 2017

\section{References}

1. Ali F, Guglin M, Vaitkevicius P, Ghali JK. Therapeutic potential of vasopressin receptor antagonists. Drugs. 2007;67:847-58.

2. Schrier RW, Gross P, Gheorghiade M, Berl T, Verbalis JG, Czerwiec FS, Orlandi C. Tolvaptan, a selective oral vasopressin V2-receptor antagonist, for hyponatremia. NEnglJMed. 2006;355:2099-112.

3. Verbalis JG, Goldsmith SR, Greenberg A, Schrier RW, Sterns RH. Hyponatremia treatment guidelines 2007: expert panel recommendations. Am J Med. 2007; 120:S1-21.

4. Loffing J, Korbmacher C. Regulated sodium transport in the renal connecting tubule (CNT) via the epithelial sodium channel (ENaC). Pflugers Arch. 2009;458: $111-35$.

5. Nielsen S, Kwon TH, Christensen BM, Promeneur D, Frokiaer J, Marples D. Physiology and pathophysiology of renal aquaporins. J Am Soc Nephrol. 1999;10:647-63.

6. Perucca J, Bichet DG, Bardoux P, Bouby N, Bankir L. Sodium excretion in response to vasopressin and selective vasopressin receptor antagonists. J Am Soc Nephrol. 2008;19(9):1721-31.

7. Wang W, Li C, Nejsum LN, Li H, Kim SW, Kwon TH, Jonassen TE, Knepper MA, Thomsen K, Frokiaer J, Nielsen S. Biphasic effects of ANP infusion in conscious, euvolumic rats: roles of AQP2 and ENaC trafficking. Am J Physiol Renal Physiol. 2006;290:F530-41. 
8. Bouley R, Breton S, Sun T, McLaughlin M, Nsumu NN, Lin HY, Ausiello DA, Brown D. Nitric oxide and atrial natriuretic factor stimulate cGMP-dependent membrane insertion of aquaporin 2 in renal epithelial cells. J Clin Invest. 2000; 106:1115-26.

9. Klokkers J, Langehanenberg P, Kemper B, Kosmeier S, von Bally G, Riethmuller C, Wunder F, Sindic A, Pavenstadt H, Schlatter E, Edemir B. Atrial natriuretic peptide and nitric oxide signaling antagonizes vasopressin-mediated water permeability in inner medullary collecting duct cells. AmJPhysiol Renal Physiol. 2009;297:F693-703.

10. Kurtz $A$, Wagner $C$. Role of nitric oxide in the control of renin secretion. Am J Physiol. 1998;275:F849-62.

11. Ortiz PA, Garvin JL. Role of nitric oxide in the regulation of nephron transport. Am J Physiol Renal Physiol. 2002;282:F777-84.

12. Al TS, Mose FH, Jensen JM, Bech JN, Pedersen EB. Effect of vasopressin antagonism on renal handling of sodium and water and central and brachial blood pressure during inhibition of the nitric oxide system in healthy subjects. BMC Nephrol. 2014;15:100.

13. Forstermann U, Sessa WC. Nitric oxide synthases: regulation and function. Eur Heart J. 2011.

14. Baylis C. Nitric oxide deficiency in chronic kidney disease. Am J Physiol Renal Physiol. 2008;294:F1-9.

15. Larsen T, Mose FH, Bech JN, Pedersen EB. Effect of nitric oxide inhibition on blood pressure and renal sodium handling: a dose-response study in healthy man. Clin Exp Hypertens. 2012;34(8):567-74.

16. Wu G, Meininger CJ. Nitric oxide and vascular insulin resistance. Biofactors. 2009;35:21-7.

17. Gansevoort RT, Arici M, Benzing T, et al. Recommendations for the use of tolvaptan in autosomal dominant polycystic kidney disease: a position statement on behalf of the ERA-EDTAWorking Groups on Inherited Kidney Disorders and European Renal Best Practice. Nephrol Dial Transplant. 2016; 31:337-48.

18. Higashihara E, Torres VE, Chapman AB, Grantham JJ, Bae K, Watnick TJ, Horie S, Nutahara K, Ouyang J, Krasa HB, Czerwiec FS. Tolvaptan in autosomal dominant polycystic kidney disease: three years' experience. Clin J Am Soc Nephrol. 2011;6:2499-507.

19. Irazabal MV, Torres VE, Hogan MC, Glockner J, King BF, Ofstie TG, Krasa HB, Ouyang J, Czerwiec FS. Short-term effects of tolvaptan on renal function and volume in patients with autosomal dominant polycystic kidney disease. Kidney Int. 2011;80:295-301.

20. Torres VE, Chapman AB, Devuyst O, Gansevoort RT, Grantham JJ, Higashihara E, Perrone RD, Krasa HB, Ouyang J, Czerwiec FS. Tolvaptan in patients with autosomal dominant polycystic kidney disease. NEnglJMed. 2012;367:2407-18.

21. Torres VE, Meijer E, Bae KT, Chapman AB, Devuyst O, Gansevoort RT, Grantham JJ, Higashihara E, Perrone RD, Krasa HB, Ouyang JJ, Czerwiec FS. Rationale and design of the TEMPO (Tolvaptan Efficacy and Safety in Management of Autosomal Dominant Polycystic Kidney Disease and its Outcomes) 3-4 Study. Am J Kidney Dis. 2011;57:692-9.

22. Berl T, Quittnat-Pelletier F, Verbalis JG, Schrier RW, Bichet DG, Ouyang J, Czerwiec FS. Oral tolvaptan is safe and effective in chronic hyponatremia. J Am Soc Nephrol. 2010;21:705-12.

23. Dasta JF, Chiong JR, Christian R, Friend K, Lingohr-Smith M, Lin J, Cassidy IB. Update on tolvaptan for the treatment of hyponatremia. Expert Rev Pharmacoecon Outcomes Res. 2012;12:399-410.

24. Elhassan EA, Schrier RW. Hyponatremia: diagnosis, complications, and management including V2 receptor antagonists. Curr Opin Nephrol Hypertens. 2011;20:161-8

25. Ellison $\mathrm{DH}$, Berl T. Clinical practice. The syndrome of inappropriate antidiuresis. NEnglJMed. 2007;356:2064-72.

26. Robertson GL. Vaptans for the treatment of hyponatremia. Nat Rev Endocrinol. 2011;7:151-61.

27. Spasovski G, Vanholder R, Allolio B, Annane D, Ball S, Bichet D, Decaux G, Fenske W, Hoorn EJ, Ichai C, et al. Clinical practice guideline on diagnosis and treatment of hyponatraemia. Nephrol Dial Transplant. 2014;29 Suppl 2:i1-39.

28. Graffe CC, Bech JN, Pedersen EB. Effect of high and low sodium intake on urinary aquaporin-2 excretion in healthy humans. AM J Physiol Renal Physiol. 2012;302(2):F264-75.

29. Matthesen SK, Larsen T, Lauridsen TG, Vase H, Gjorup PH, Nykjaer KM, Nielsen S, Pedersen EB. Effect of amiloride and spironolactone on renal tubular function, ambulatory blood pressure, and pulse wave velocity in healthy participants in a double-blinded, randomized, placebo-controlled, crossover trial. Clin Exp Hypertens. 2012;34(8):588-600.
30. Pedersen RS, Bentzen $H$, Bech JN, Pedersen EB. Effect of water deprivation and hypertonic saline infusion on urinary AQP2 excretion in healthy humans. Am J Physiol Renal Physiol. 2001;280(5):F860-7.

31. Hager H, Kwon TH, Vinnikova AK, Masilamani S, Brooks HL, Frøkiaer J, Knepper MA, Nielsen S. Immunocytochemical and immunoelectron microscopic localization of alpha-, beta-, and gamma-ENaC in rat kidney. Am J physiol Renal Physiol. 2001;280(6):F1093-106.

32. Pedersen EB, Danielsen $H$, Spencer ES. Effect of indapamide on renal plasma flow, glomerular filtration rate and arginine vasopressin in plasma in essential hypertension. Eur J Clin Pharmacol. 1984;26(5):543-7.

33. Pedersen EB, Eiskjaer $H$, Madsen B, Danielsen $H$, Egeblad M, Nielsen CB. Effect of captopril on renal extraction of renin, angiotensin II, atrial natriuretic peptide and vasopressin, and renal vein renin ratio in patients with arterial hypertension and unilateral renal artery disease. Nephrol Dial Transplant. 1993; 8(10):1064-70

34. Shoaf SE, Bricmont P, Mallikaarjun S. Absolute bioavailability of tolvaptan and determination of minimally effective concentrations in healthy subjects. Int J Clin Pharmacol Ther. 2012;50:150-6.

35. Shoaf SE, Wang Z, Bricmont P, Mallikaarjun S. Pharmacokinetics, pharmacodynamics, and safety of tolvaptan, a nonpeptide AVP antagonist, during ascending single-dose studies in healthy subjects. J Clin Pharmacol. 2007:47:1498-507.

36. Bhatt PR, McNeely EB, Lin TE, Adams KF, Patterson JH. Review of tolvaptan's pharmacokinetic and pharmacodynamic properties and drug interactions. J Clin Med. 2014;3(4):1276-90.

37. Blanchard A, Frank M, Wuerzner G, Peyrard S, Bankir L, Jeunemaitre X, Azizi $M$. Antinatriuretic effect of vasopressin in humans is amiloride sensitive, thus ENaC dependent. Clin J Am Soc Nephrol. 2011;6(4):753-9.

38. Lauridsen TG, Vase $H$, Bech JN, Nielsen S, Pedersen EB. Direct effect of methylprednisolone on renal sodium and water transport via the principal cells in the kidney. Eur J Endocrinol. 2010;162:961-9.

39. Matthesen SK, Larsen T, Vase H, Lauridsen TG, Pedersen EB. Effect of potassium supplementation on renal tubular function, ambulatory blood pressure and pulse wave velocity in healthy humans. Scand J Clin Lab Invest. 2012;72(1):78-86

\section{Submit your next manuscript to BioMed Central and we will help you at every step:}

- We accept pre-submission inquiries

- Our selector tool helps you to find the most relevant journal

- We provide round the clock customer support

- Convenient online submission

- Thorough peer review

- Inclusion in PubMed and all major indexing services

- Maximum visibility for your research

Submit your manuscript at www.biomedcentral.com/submit
Biomed Central 